\title{
SARS-Cov-2 Infection Uncovering Latent Mycobacterium Leprae Infection
}

Christopher Mancuso, DO, MHS'1, Paloma Reiter, DO'1, Regina Zambrano, DO², Ritu Saini, $\mathrm{MD}^{1}$, Suzanne Sirota-Rozenberg, $\mathrm{DO}^{1}$

${ }^{1}$ St. John's Episcopal Hospital, Department of Dermatology, Far Rockaway, New York

2 St. John's Episcopal Hospital, Department of Family Medicine, Far Rockaway, New York

\section{ABSTRACT}

Leprosy is a chronic granulomatous infection caused by the bacteria Mycobacterium leprae. It can remain dormant in a patient without any symptoms until triggered by a stressor on the body. During the coronavirus disease 2019 (COVID-19) pandemic, a significant amount of emotional and psychological stress was endured by patients. In addition, many patients experienced exacerbations of their preexisting conditions. Few reports have described cases of leprosy with concurrent COVID19. This brief article presents a case of newly diagnosed leprosy in a middle-aged male following a COVID-19 infection. To our knowledge, this is the first reported case of its kind in the United States.

\section{INTRODUCTION}

As the number of coronavirus disease 2019 (COVID-19) cases rise globally, more information is being brought to light regarding the relationship between COVID19 and leprosy. There are very few reports of leprosy with concurrent COVID-19. ${ }^{1}$ Limited literature suggests two theories have been postulated thus far: (1) COVID-19 infection may trigger a leprosy reaction; and (2) leprosy treatment may cause patients to experience a severe manifestation of COVID-19 infection. ${ }^{1,2}$

Also known as Hansen's disease, leprosy is a chronic granulomatous infection caused by the bacteria Mycobacterium leprae that spreads via respiratory droplets. ${ }^{3}$ Leprosy involves immune factors, such as interleukins (IL) 4, 5, 6, 10, 13, that COVID19 may activate or exacerbate. ${ }^{4,5}$ In addition, infections and stress are thought to be triggers for leprosy reactions. ${ }^{6}$ While early diagnosis and treatment with antibiotics and anti-inflammatory medications can cure an individual with leprosy, it may create susceptibility to COVID-19 by changing a patient's cytokine profile. ${ }^{1,5,7,8}$ Despite the current social distancing mandates to prevent acquisition of COVID-19, many patients with leprosy are a part of lower socioeconomic groups associated with overcrowded living and poor sanitary conditions. ${ }^{1,3}$ Combined with the historical shame and discrimination that leprosy patients experience, this creates major vulnerability in this patient population. ${ }^{1,3,6}$ We present a case of newly diagnosed leprosy post-COVID-19 infection in a middleaged male. To our knowledge, this is the first reported case of its kind in the United States.

\section{CASE REPORT}

(c) 2022 THE AUTHORS. Published by the National Society for Cutaneous Medicine. 
A 46-year-old Fitzpatrick VI, AfricanAmerican man with a significant past medical history of recent COVID-19 infection in April 2020 presented to the dermatology clinic on July 2, 2020 with a three-week history of a rash on his face. The patient reported that he migrated from Guyana three years ago and did not have a history of or exposure to leprosy. Of note, the patient did admit to an increased amount of stress amidst the COVID-19 pandemic. On physical examination, the patient had multiple edematous and erythematous plaques of his forehead, periocular areas, nose, malar cheeks, and temples; forming the classical leonine facies (Figure 1). Patient admitted to hyperesthesia of the upper extremities without pruritus. A $3 \mathrm{~mm}$ punch biopsy was performed on a plaque of the patient's left upper cheek. The biopsy was reported as multibacillary leprosy with innumerable beaded or fragmented organisms (Figure 2). The patient was started on Clofazimine $50 \mathrm{mg}$ daily and Rifampin $600 \mathrm{mg}$ daily, and sent for glucose6-phosphate dehydrogenase (G6PD) deficiency testing before starting Dapsone $100 \mathrm{mg}$ daily. Infectious disease was consulted for further management and the Health Department was notified.

\section{DISCUSSION}

COVID-19 is a viral respiratory infection caused by severe acute respiratory syndrome coronavirus 2 (SARS-CoV-2) that emerged in Wuhan, China in December 2019. The spectrum of disease caused by
COVID-19 ranges from asymptomatic to SARS. ${ }^{7}$ As the number of cases increase worldwide, clinicians realize that patients with COVID-19 may experience various coinfections. Presently, knowledge regarding co-infection with leprosy is sparse, but a few theories have been proposed.

Leprosy is a chronic granulomatous infection that leads to various cutaneous manifestations. . $^{3,5}$ Type 1 leprosy, the tuberculoid form, is a Type IV hypersensitivity reaction with a TH1 cellmediated response..$^{3,4,5}$ This form is characterized by an intense TNF- $\alpha$ and IFNy reaction, leading to decreased $M$. leprae bacilli and less cutaneous manifestations. . $^{3,5}$ Type 2 leprosy, the lepromatous form, is a Type III hypersensitivity reaction with a $\mathrm{TH} 2$ helper T-cell response. . $^{3,45}$ This form demonstrates impaired immunity due to IL-4, IL-10, and TGF- $\beta$ secretion, causing large numbers of bacilli and destruction of peripheral nerves, skin, mucosa, and bones. ${ }^{3,4,5}$ Type 2 reactions, which include erythema nodosum leprosum, are associated with immune complex deposition and neutrophil infiltrate. ${ }^{3}$ It has been suggested that microbial infections and stress are triggers for leprosy reactions. The literature has shown SARSCoV-2 to influence neutrophil infiltration, eventually creating hyperinflammation or cytokine storm syndrome in a number of COVID-19 cases. $^{2,4,9,10}$ Limited studies proposed two theories to explain the relationship between leprosy and COVID-19. 


\section{SKIN}

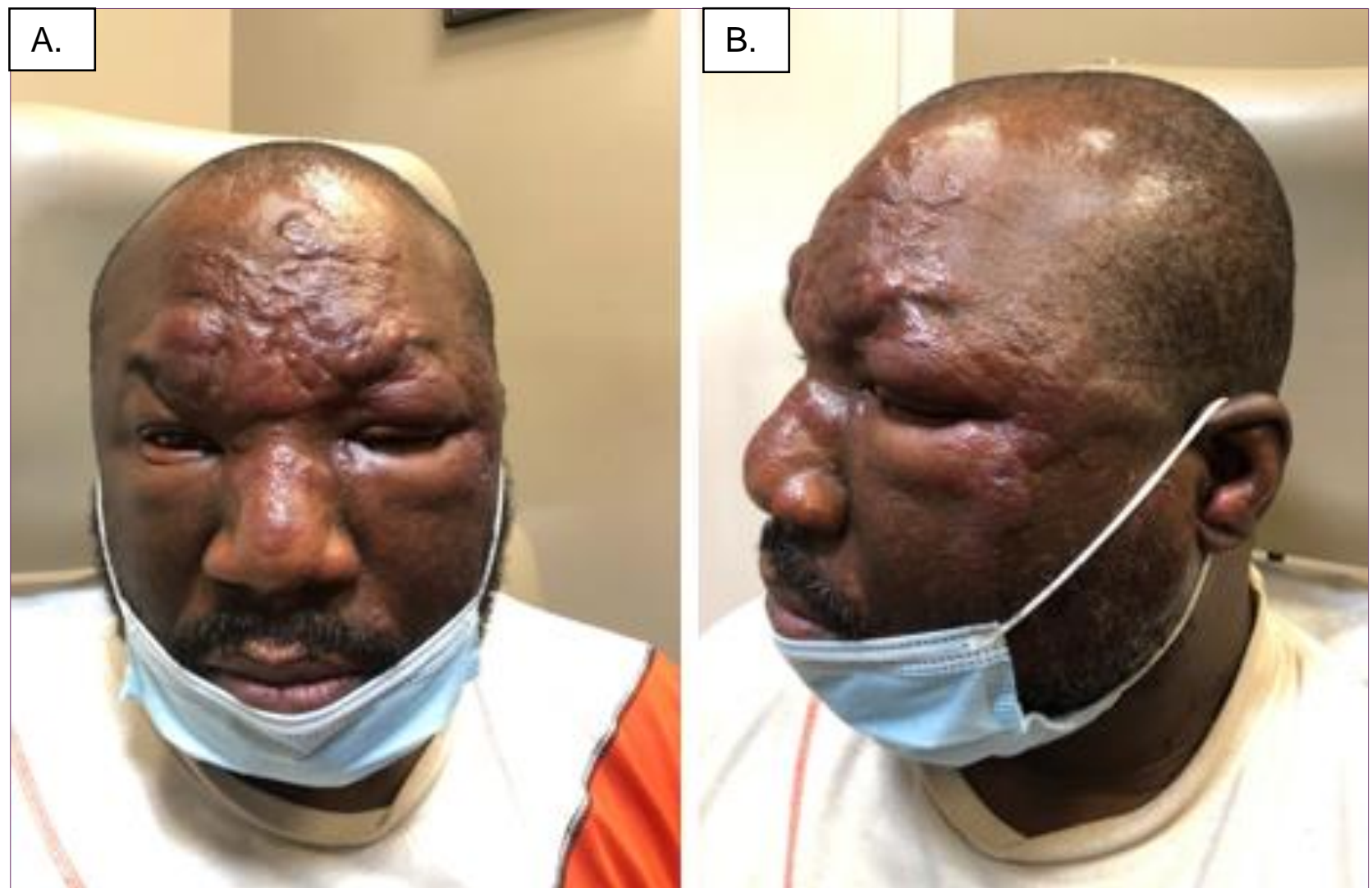

Figure 1. A) Cushion-like centrofacial infiltrates (leonine facies) of the forehead, loss of eyebrows and eyelashes, distortion of the nose B) Leonine facies with thickening of the temple, and characteristic infiltration of the auricle

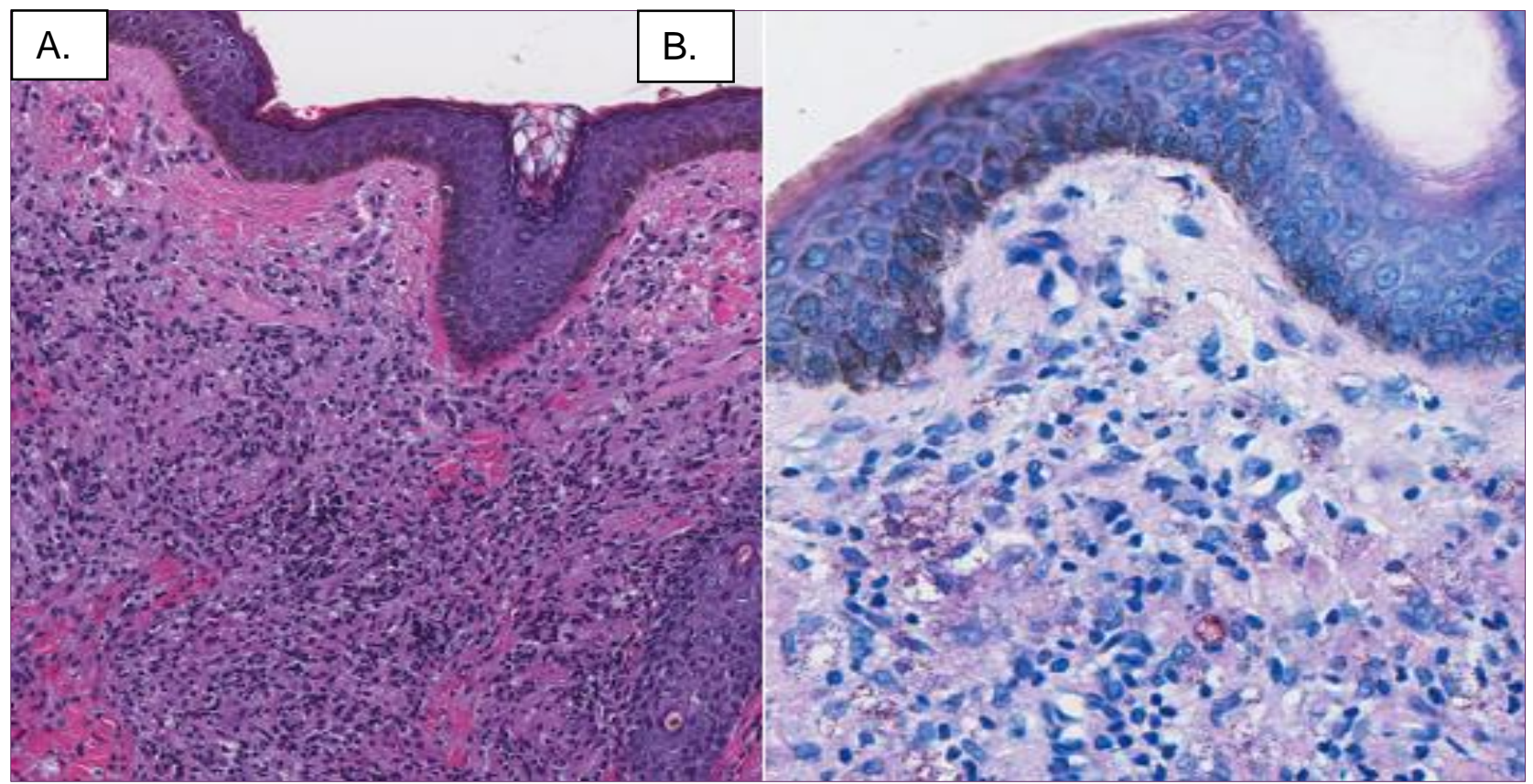

Figure 2. A) Hematoxylin-eosin stain section of biopsy demonstrating nodular mononucleated histiocytes with numerous admixed lymphocytes and a grenz zone within the papillary dermis (100x magnification). B) Fite stain section of biopsy notable for numerous beaded or fragmented acid-fast bacilli with a perivascular lymphohistiocytic infiltrate and globi (400x magnification) 
The first theory states that COVID-19 is a risk factor for triggering a leprosy reaction due to its effect on various immunologic events. Type 1 leprosy involves multiple cytokines, therefore COVID-19 infection may trigger a Type 1 leprosy reaction due to its ability to induce secretion of similar cytokines. Type 2 leprosy is known to be associated with dermal neutrophil infiltrate, leading to skin disfiguration. SARS-CoV-2 also induces extensive neutrophil infiltrate, specifically in the pulmonary capillaries, that eventually enters circulation. ${ }^{1,9,10}$ Increased circulating neutrophils influenced by SARSCoV-2 may potentiate or exacerbate a Type 2 leprosy infection, as well as hasten or worsen clinical course. ${ }^{2}$ Overall, this first theory suggests that COVID-19 may trigger lepromatous reactions.

The second theory proposes that leprosy patients undergoing active treatment with anti-inflammatory medications are at an increased risk of acquiring severe manifestations of COVID-19. ${ }^{1,7}$ Specifically, Type 1 leprosy is treated with prednisone, and Type 2 leprosy is treated with thalidomide. ${ }^{1,5}$ Both drugs interfere with the host's inflammatory response and create a dose-dependent immunosuppressed state. ${ }^{8}$ There is concern that patients being treated for leprosy are vulnerable to a severe clinical manifestation of COVID-19, such as SARS. ${ }^{1,7,8}$ There is also conflicting literature that suggests immunosuppressed patients may instead be protected. ${ }^{7}$ Currently, more large-scale studies must be done to state with confidence how immunosuppressives impact the acquisition and clinical course of COVID-19. Although this theory does not relate to our case directly, it is an important consideration for the management of leprosy patients during the pandemic.

\section{CONCLUSION}

Leprosy reactions may be triggered by stress and infections, and the COVID-19 pandemic has caused significant emotional and psychological stress on patients. ${ }^{6}$ When considering this and the two theories proposed, our unique case suggests that COVID-19 may induce or exacerbate leprosy reactions. ${ }^{4}$ Leprosy reactions may lead to disfiguring complications and social isolation in a vulnerable patient population, therefore early diagnosis and treatment is necessary to minimize detrimental complications. ${ }^{6}$ Throughout the pandemic, dermatologists must increase their index of suspicion for uncommon cases due to the possibility of COVID-19 triggers.

Acknowledgements: We would like to thank Shane A. Meehan, MD, Director of Dermatopathology at NYU Langone Health for his assistance with this case and for the histologic photographs.

\section{Conflict of Interest Disclosures: None}

Funding: None

Corresponding Author:

Christopher Mancuso

190 Beach 69th Street, Apt 2E

Arverne, NY 11692

Email:cjmancuso@gmail.com

\section{References:}

1. Rathod S, Suneetha S, Narang T, et al. Management of Leprosy in the Context of COVID-19 Pandemic: Recommendations by SIG Leprosy (IADVL Academy). Indian Dermatol Online J. 2020;11(3):345-348. Published 2020 May 10. doi:10.4103/idoj.IDOJ_234_20

2. Schmitz V, Dos Santos JB. COVID-19, leprosy, and neutrophils. PLoS Negl Trop Dis. 2021;15(1):e0009019. Published 2021 Jan 7. doi:10.1371/journal.pntd.0009019itz 
3. Antunes DE, Goulart IMB, Goulart LR. Will cases of leprosy reaction increase with COVID-19 infection? PLoS Negl Trop Dis. 2020;14:e0008460 10.1371/journal.pntd.0008460

4. Santos VS, Quintans-Júnior LJ, Barboza WS, Araújo AAS, Martins-Filho PR. Clinical characteristics and outcomes in patients with COVID-19 and leprosy [published online ahead of print, $2020 \mathrm{~A}$

5. Nery JA, Bernardes Filho F, Quintanilha J, Machado AM, Oliveira Sde S, Sales AM. Understanding the type 1 reactional state for early diagnosis and treatment: a way to avoid disability in leprosy. An Bras Dermatol. 2013;88(5):787-792. doi:10.1590/abd18064841.20132004

6. Bilik L, Demir B, Cicek, D. Leprosy Reactions. Hansen's Disease - The Forgotten and Neglected Disease. 2019. doi: 10.5772/intechopen.72481

7. Thng ZX, De Smet MD, Lee CS, et al. COVID-19 and immunosuppression: a review of current clinical experiences and implications for ophthalmology patients taking immunosuppressive drugs [published online ahead of print, 2020 Jun 12]. Br J Ophthalmol. 2020;bjophthalmol-2020-316586.

doi:10.1136/bjophthalmol-2020-316586

8. Liu D, Ahmet A, Ward L, et al. A practical guide to the monitoring and management of the complications of systemic corticosteroid therapy. Allergy Asthma Clin Immunol. 2013;9(1):30.

Published 2013 Aug 15. doi:10.1186/1710-14929-30

9. Barnes BJ, Adrover JM, Baxter-Stoltzfus A, et al. Targeting potential drivers of COVID-19: Neutrophil extracellular traps. J Exp Med. 2020;217(6):e20200652. doi:10.1084/jem.20200652

10. Mehta P, McAuley DF, Brown M, et al. COVID19: consider cytokine storm syndromes and immunosuppression. Lancet. 2020;395(10229):1033-1034. doi:10.1016/S0140-6736(20)30628-0 